\title{
HARDER-NARASIMHAN REDUCTION OF A PRINCIPAL BUNDLE
}

\author{
INDRANIL BISWAS AND YOGISH I. HOLLA
}

\begin{abstract}
Let $E$ be a principal $G$-bundle over a smooth projective curve over an algebraically closed field $k$, where $G$ is a reductive linear algebraic group over $k$. We construct a canonical reduction of $E$. The uniqueness of canonical reduction is proved under the assumption that the characteristic of $k$ is zero. Under a mild assumption on the characteristic, the uniqueness is also proved when the characteristic of $k$ is positive.
\end{abstract}

\section{$\S 1$. Introduction}

Let $X$ be a connected smooth projective curve defined over an algebraically closed field $k$. A vector bundle $E$ over $X$ is called semistable if for every subbundle $F$ of $E$, the inequality

$$
\frac{\text { degree }(F)}{\operatorname{rank}(F)} \leq \frac{\text { degree }(E)}{\operatorname{rank}(E)}
$$

is valid [4]. Given any vector bundle $E$, there is a unique filtration of $E$

$$
0=F_{0} \subset F_{1} \subset F_{2} \subset \cdots \subset F_{l-1} \subset F_{l}=E
$$

by subbundles which is characterized by the two conditions that all $F_{i} / F_{i-1}$, $i \in[1, l]$, are semistable and the quotients degree $\left(F_{i} / F_{i-1}\right) / \operatorname{rank}\left(F_{i} / F_{i-1}\right)$ are strictly decreasing as $i$ increases. This filtration was introduced in [4] and it is known as the Harder-Narasimhan filtration or the canonical filtration.

Let $G$ be a connected reductive linear algebraic group over $k$. A principal $G$-bundle over $X$ is a variety $E$ equipped with a right action of $G$ and a $G$-invariant smooth projection $\pi: E \longrightarrow X$ such that the map $E \times_{X}(X \times G) \longrightarrow E \times_{X} E$ of fiber products over $X$ defined by $(y,(x, g)) \longmapsto(y, y g)$ is an isomorphism, where $x \in X, y \in \pi^{-1}(x)$ and $g \in G$. The above notion of semistability of a vector bundle extends to $G$ bundles (see Section 2 for the definition).

Received September 25, 2002.

2000 Mathematics Subject Classification: 14H60, 32L05. 
Let $E_{G}$ be a principal $G$-bundle over $X$ and $E_{P} \subset E_{G}$ a reduction of structure group of $E_{G}$ to a parabolic subgroup $P$ of $G$. In [9], Ramanathan calls this reduction to be canonical if the following two conditions hold:

(1) if $L$ is the Levi factor of $P$, then the principal $L$-bundle $E_{P} \times{ }_{P} L$ obtained by extending the structure group is semistable (recall that $L$ is a quotient of $P$ );

(2) after fixing a Borel subgroup $B \subset P$ of $G$, if we take any nontrivial character $\chi$ of $P$ which is a nonnegative linear combination of simple roots, then the associated line bundle $\left(E_{P} \times k\right) / P$ over $X$ is of positive degree.

For $G=\mathrm{GL}(n, k)$, a reduction $E_{P}$, where $P$ is a parabolic subgroup, gives a filtration of the rank $n$ vector bundle associated to $E$ by the standard representation. It is easy to see that $E_{P}$ is canonical in the above sense if and only if the corresponding filtration of the associated vector bundle coincides with its Harder-Narasimhan filtration. In [9] Ramanathan asserted, without proof, that any principal $G$-bundle admits a unique canonical reduction.

The aim here is to present a simple bundle theoretic proof of the existence and uniqueness of the canonical reduction for principal $G$-bundles over $X$. We construct a canonical reduction of any given $G$-bundle (Proposition 3.1). We show that the canonical reduction is unique under the assumption that the characteristic of $k$ is zero (Theorem 4.1). As an outcome, we are able to describe the behavior of the canonical reduction with respect to extensions of structure group by faithful homomorphism (Theorem 5.1).

An analog of the Harder-Narasimhan filtration for principal $G$-bundles was first formulated by Atiyah and Bott in [1]. More precisely, given a principal bundle over a compact Riemann surface, they considered the HarderNarasimhan filtration of the adjoint vector bundle associated to it for the adjoint action of $G$ on its Lie algebra. In [1] it is shown that the HarderNarasimhan filtration is symmetric, has an odd number of terms, and the middle term in the filtration gives a reduction of structure group of the principal $G$-bundle to a parabolic subgroup.

This raises two issues:

(1) to prove the assertion in [9], and

(2) to prove that the canonical reduction, in the sense of [9], coincides with the reduction given in [1].

The first proof of the existence and uniqueness of the canonical reduction was given by Behrend for the case when $X$ is a smooth projective curve [3]. The proof of Behrend works in all characteristics for reductive group 
schemes over a curve. His main argument involves key usage of the complementary polyhedron. However it may be desirable to have a proof which is more bundle theoretic.

On the other hand, for $k=\mathbb{C}$, existence and uniqueness of canonical reduction of a principal $G$-bundle over a compact Kähler manifold was established in [2]. In this set-up it was also proved that the canonical reduction coincides with the one given by [1].

Subsequently, in [7], the equivalence between the two different formulations of Harder-Narasimhan reduction, namely the one given in [1] and the one in [9], for principal $G$-bundles over a connected smooth projective curve over an algebraically closed field of suitably positive characteristic was established. The method of [7] is quite similar to the proof in [2] that the two formulations of the Harder-Narasimhan reductions are equivalent.

In the final section we address the case of positive characteristic using the method for characteristic zero. The construction of canonical reduction requires the characteristic to be suitably positive. However, it is possible to give a slightly better bound than in [7] for the range of characteristics where the Harder-Narasimhan reduction is valid (Corollary 6.11). We also describe the behavior of the canonical filtrations with respect to extension of structure groups by faithful homomorphisms under an assumption on the characteristic (see Theorem 6.13).

The proofs extend to all dimensions of $X$ once we extend to the notion of reduction of structure group to those defined over the complement of a closed subvariety of codimension at least two.

\section{$\S 2$. Preliminaries}

Let $X$ be a connected smooth projective curve over an algebraically closed field $k$ of arbitrary characteristic. Let $G$ be a connected reductive algebraic group over $k$.

Take a principal $G$ bundle $E$ over $X$. So, in particular, $G$ acts freely on the right of $E$ with $X$ as the quotient. For any quasi-projective scheme $F$ on which $G$ operates on the left, consider the action of $G$ on $E \times F$ defined by $g \circ\left(e^{\prime}, f^{\prime}\right)=\left(e^{\prime} g, g^{-1} f^{\prime}\right)$, where $g \in G, e^{\prime} \in E$ and $f^{\prime} \in F$. The quotient $(E \times F) / G$ for this action, which is a fiber bundle over $X$, will be denoted by $E(F)$. For any subgroup $H$ of $G$, we denote the fiber bundle $E(G / H)$ over $X$, defined using the obvious left action of $G$ on $G / H$, by $E / H$. Also, for any $G$-equivariant morphism

$$
f: F_{1} \longrightarrow F_{2}
$$

we obtain an associated morphism $f_{E}: E\left(F_{1}\right) \longrightarrow E\left(F_{2}\right)$. It is easy to see that $f_{E}$ is a closed embedding if $f$ is so. 
Let $\rho: G \longrightarrow G^{\prime}$ be a group homomorphism of algebraic groups. Consider the action of $G$ on $G^{\prime}$ defined by left-multiplication. Then the fiber bundle $E\left(G^{\prime}\right)$ is a principal $G^{\prime}$ bundle over $X$. The $G^{\prime}$-bundle $E\left(G^{\prime}\right)$ is known as the one obtained from $E$ by extending the structure group to $G^{\prime}$ using $\rho$. We will denote $E\left(G^{\prime}\right)$ by $\rho_{*} E$.

Let $H$ be any closed subgroup of $G$. A pair $\left(E^{\prime}, \phi\right)$, where $E^{\prime}$ is a principal $H$-bundle and $\phi: i_{*} E^{\prime} \longrightarrow E$ is an isomorphism of principal $G^{-}$ bundles, is said to give a reduction of structure group of $E$ to $H$. Reductions of structure group of $E$ to $H$ and sections of the fiber bundle $E / H \longrightarrow X$ are clearly in bijective correspondence. Indeed, $E$ is a principal $H$-bundle over $E / H$. The bijective correspondence sends a section $\sigma: X \longrightarrow E / H$ to the principal $H$-bundle $\sigma^{*} E$. So, by a slight abuse of notation, we will denote by $(H, \sigma)$ a reduction of structure group to $H$.

Lemma 2.1. Let $E$ be a principal $G$-bundle on $X$ and $\rho: G \longrightarrow G_{1}$ be a surjective homomorphism. Let $H_{1}$ be a closed subgroup of $G_{1}$. If the principal $G_{1}$-bundle $\rho_{*} E$ admits a reduction of structure group $\sigma_{1}$ to $H_{1}$ then the principal $G$-bundle $E$ admits a reduction of structure group $\sigma$ to $H=\rho^{-1}\left(H_{1}\right)$ such that $\rho^{\prime}{ }_{*} \sigma^{*} E=\sigma_{1}^{*} \rho_{*} E$, where $\rho^{\prime}=\left.\rho\right|_{H}$.

Proof. Since $G / H \cong G_{1} / H_{1}$, a reduction of structure group of $\rho_{*} E$ to $H_{1}$ automatically gives rise to a reduction of structure group of $E$ to $H$.

For any $g \in G$, let $R_{g}: E \longrightarrow E$ be the action of $g$ on $E$. Then $R_{g}$ induces a morphism $E / H \longrightarrow E / g^{-1} H g$. Hence any reduction $\sigma$ of structure group to $H$ gives rise to a reduction $\sigma_{g}$ of structure group to $g^{-1} \mathrm{Hg}$. More precisely, $\sigma_{g}$ is the composition of $\sigma$ with the isomorphism $E / H \longrightarrow E / g^{-1} H g$ obtained using $R_{g}$. We say that these two reductions are equivalent.

Let $\mathfrak{g}$ and $\mathfrak{h}$ denote the Lie algebras of $G$ and $H$ respectively. Consider the adjoint action of a Lie group on its Lie algebra. We denote the vector bundle $E(\mathfrak{g})$ over $X$ by $\operatorname{ad}(E)$, which is also known as the adjoint vector bundle. Then for a reduction of structure group $\sigma$ of $E$ to $H$, we evidently have $\sigma^{*} E(\mathfrak{h})=\operatorname{ad}\left(\sigma^{*} E\right)$.

The conjugation action of $G$ on itself will be denoted by $c$. More precisely, $c_{g}(h):=g h g^{-1}$. The associated fiber bundle $E(G)$ for the conjugation action is a group scheme over $X$, which is known as the adjoint bundle. Its Lie algebra is clearly the adjoint vector bundle $E(\mathfrak{g})$ over $X$.

Suppose $H_{1}$ and $H_{2}$ are closed connected subgroups of $G$. Let $\sigma_{1}$ and $\sigma_{2}$ be reductions of structure group of $E$ to $H_{1}$ and $H_{2}$ respectively. To this data we can associate sub-group schemes $\sigma_{i}^{*} E\left(H_{i}\right)$ of $E(G)$ and their Lie 
algebras $\operatorname{ad}\left(\sigma_{i}^{*} E\right)$, which are sub-bundles of $\operatorname{ad}(E)$. The following lemma gives a way of understanding the reduction of structure group using the Lie algebra $\operatorname{ad}(E)$.

Lemma 2.2. Suppose the two sub-bundles $\operatorname{ad}\left(\sigma_{1}^{*} E\right)$ and $\operatorname{ad}\left(\sigma_{2}^{*} E\right)$ of $\operatorname{ad}(E)$ coincide. Then there exists an element $g \in G$ such that $H_{1}=$ $g^{-1} H_{2} g$. Moreover, if the normalizer of $H_{1}$ in $G$ is $H_{1}$ itself then the two reductions $\sigma_{1}^{*} E$ and $\sigma_{2}^{*} E$ coincide, or in other words, the reductions $\sigma_{1}$ and $\sigma_{2}$ are equivalent.

Proof. We first prove that the two group schemes $\sigma_{1}^{*} E\left(H_{1}\right)$ and $\sigma_{2}^{*} E\left(H_{2}\right)$ are the same. As the question is local on the base, we may assume that $X=\operatorname{Spec}(A)$ is an integral affine scheme with function field $K$.

Using a descent argument (by going to a faithfully flat cover) we may assume that the group schemes $\sigma_{i}^{*} E\left(H_{i}\right)$ and $E(G)$ are products $X \times H_{i}$ and $X \times G$ respectively.

Now we also have $E(G)=\operatorname{Spec}(B)$ and the group schemes $\sigma_{i}^{*} E\left(H_{i}\right)$ are defined by ideals $I_{i}$. If we base change to function field, we get two closed connected subgroups with the property that the Lie algebra of one is contained in the other. Hence we obtain that $I_{2} \otimes_{A} K \subset I_{1} \otimes_{A} K$. Since the ideals $I_{i}$ are finitely generated prime ideals it follows immediately that there is an element $f \in A$ such that $f I_{2} \subset I_{1}$. Now since $B$ is a tensor product of $A$ and $k[G]$ and the ideals $I_{i}$ are also tensor products of $A$ and ideals in $k[G]$, we conclude that $I_{2} \subset I_{1}$. Similarly one obtains the opposite inclusion. This proves the equality

$$
\sigma_{1}^{*} E\left(H_{1}\right)=\sigma_{2}^{*} E\left(H_{2}\right)
$$

of sub-group schemes of $E(G)$.

To prove the first assertion, we compare the fibers of the above group schemes. Let $x \in X$ be a point. Observe that the fibers of $E(G)$ and $\sigma_{i}^{*} E\left(H_{i}\right)$ at $x$ are of the form $\left(E_{x} \times G\right) / G$ and $\left(\sigma_{i}^{*} E_{x} \times H_{i}\right) / H_{i}$ respectively. For $i=1,2$, let $e_{i} \in\left(\sigma_{i}^{*} E\right)_{x}$ be a rational point. Then there is a unique element $g \in G$ with the property that $g\left(e_{1}\right)=e_{2}$.

We claim that $H_{1}=g^{-1} H_{2} g$. To see this first note that it follows from the equality (1) that given an element $\left(e_{1}, h_{1}\right) \in \sigma_{1}^{*} E \times H_{1}$ there is an element $g_{1} \in H_{1}$ such that $\left(g_{1}\left(e_{1}\right), c_{g_{1}}\left(h_{1}\right)\right) \in \sigma_{2}^{*} E \times H_{2}$. Consequently, there is an element $h \in H_{2}$ such that $h\left(g_{1}\left(e_{1}\right)\right)=e_{2}$. This implies that $h g_{1}=g$, and hence we have proved that $H_{1} \subset g^{-1} H_{2} g$. Similarly, we have $H_{2} \subset g H_{1} g^{-1}$. Therefore, $H_{1}=g^{-1} H_{2} g$. 
In order to show that the reductions are equivalent we need to show that the element $g$ as defined above has the property that for any $x^{\prime} \in X$ the equality $g\left(\left(\sigma_{1}^{*} E\right)_{x^{\prime}}\right)=\left(\sigma_{2}^{*} E\right)_{x^{\prime}}$ is valid. Just as above, by choosing rational points $e_{i}^{\prime}$ in $\left(\sigma_{i}^{*} E\right)_{x^{\prime}}$ we get a unique element $g^{\prime} \in G$ such that $g^{\prime}\left(e_{1}^{\prime}\right)=e_{2}^{\prime}$ and $c_{g^{\prime}}\left(H_{1}\right)=H_{2}$. Therefore, we have $c_{g^{\prime-1} g}\left(H_{1}\right)=H_{1}$, as $H_{1}=g H_{2} g^{-1}$. Since the normalizer of $H_{1}$ in $G$ is given to be $H_{1}$ itself, we conclude that $g=g^{\prime} h$ for some $h \in H_{1}$. Finally, since

$$
g\left(\left(\sigma_{1}^{*} E\right)_{x^{\prime}}\right)=g^{\prime} h\left(\left(\sigma_{1}^{*} E\right)_{x^{\prime}}\right)=g^{\prime}\left(\left(\sigma_{1}^{*} E\right)_{x^{\prime}}\right)=\left(\sigma_{2}^{*} E\right)_{x^{\prime}}
$$

the proof of the lemma is complete.

Remark 2.3. The proof shows more generally that if we are only given the inclusion $\operatorname{ad}\left(\sigma_{1}^{*} E\right) \subset \operatorname{ad}\left(\sigma_{2}^{*} E\right)$, then firstly we have an inclusion of the group schemes $\sigma_{1}^{*} E\left(H_{1}\right) \subset \sigma_{2}^{*} E\left(H_{2}\right)$, and secondly, we can also find an element $g \in G$ such that $H_{1} \subset g H_{2} g^{-1}$.

The transition from the group schemes to principal bundles can also be made as follows.

Lemma 2.4. Suppose that $E$ is a principal $G$-bundle. Let $H$ be a closed connected subgroup of $G$. Suppose the normalizer of $H$ in $G$ is $H$ itself. Let $\mathcal{H} \subset E(G)$ be a subgroup scheme over $X$ of the adjoint bundle whose fibers are of the type $H$ (that is, each geometric fiber is a conjugate of $H$ ). Then there is a reduction of structure group $\sigma$ of the $G$-bundle $E$ to $H$ such that $\sigma^{*} E(H)=\mathcal{H}$.

Proof. Consider the natural projection $q: E \times G \longrightarrow(E \times G) / G=$ $E(G)$. Let

$$
E_{H} \subset E
$$

be consisting of all points $y$ such that $q(y, g) \in \mathcal{H}$ for every $g \in H$. For the action of $G$ on $E$, the sub-variety $E_{H}$ is preserved by the action of $H$. Since the normalizer of $H$ in $G$ is $H$ itself, the action of $H$ on the fibers of $E_{H}$ is transitive. Therefore, we have reduction of structure group of $E$ to $H$, completing the proof of the lemma.

We recall the definition of semistability of principal $G$-bundles. A principal $G$-bundle $E$ is called semistable if for any reduction of structure group $(P, \sigma)$ to a parabolic $P$, where $\sigma: X \longrightarrow E / P$ is a section, we have

$$
\operatorname{degree}\left(\sigma^{*} T_{E / P}\right) \geq 0 .
$$


Here $T_{E / P}$ is the relative tangent bundle for the projection $E / P \longrightarrow X$. The above condition is equivalent to the following. For any reduction $(P, \sigma)$ to a parabolic subgroup $P$ and for any dominant character $\chi$ of $P$, trivial on the center of $G$, the associated line bundle $\chi_{*} \sigma^{*} E$ has nonpositive degree.

Now we state a lemma which will be used later.

LEMMA 2.5. Let $E$ be a principal $G$-bundle such that $\operatorname{ad}(E)$ is a semistable vector bundle. Then $E$ is semistable.

Proof. If $(P, \sigma)$ is a reduction, then the vector bundle $\sigma^{*} T_{E / P}$ is a quotient of $\operatorname{ad}(E)$. Since $\operatorname{ad}(E)$ is semistable of degree zero, we have degree $\left(\sigma^{*} T_{E / P}\right) \geq 0$.

The converse of Lemma 2.5 is also true in characteristic 0 , as shown in the proof of Theorem 4.1 .

\section{$\S 3$. The existence of canonical reduction}

The aim in this section is to show that the canonical reduction exists for any characteristic.

By a parabolic subgroup of $G$ we will mean a closed algebraic subgroup $P$ such that $G / P$ is complete. In particular, $G$ itself is considered as a parabolic subgroup of $G$.

Recall from [9] that for a principal $G$-bundle $E$, a reduction of structure group $(P, \sigma)$ of $E$ to a parabolic subgroup $P$ of $G$ is called canonical if the following two conditions hold

(1) The Levi bundle $E_{L}$ associated, by extension of structure group, to $\sigma^{*} E$ for the projection $P \longrightarrow L$ is semistable.

(2) For every non-trivial character $\chi$ of $P$ which is a nonnegative linear combination of simple roots with respect to some Borel subgroup contained in $P$, the line bundle $\chi_{*} \sigma^{*} E$ on $X$ has positive degree.

As remarked in the introduction, for $G=\mathrm{GL}(n, k)$ this corresponds precisely to the Harder-Narasimhan filtration of the associated vector bundle of rank $n$.

For a principal $G$-bundle $E$, define the integer

$$
d_{E}=\min \left\{\operatorname{degree}\left(\sigma^{*} T_{E / P}\right) \mid(P, \sigma) \text { is a reduction as above }\right\},
$$

where $T_{E / P}$, as before, is the relative tangent bundle. It should be emphasized that $d_{E}$ is defined by taking the minimum of all possible pairs $(P, \sigma)$. In particular, $P$ is not fixed.

Since $\sigma^{*} T_{E / P}$ is a quotient of $\operatorname{ad}(E)$, using the fact that quotients of a fixed bundle have their degrees bounded from below we obtain that $d_{E}$ is well defined as an integer.

The following proposition proves the existence of a canonical reduction. 
Proposition 3.1. Let $(P, \sigma)$ be a reduction of structure group of $E$ to $P$ such that the following two conditions are valid:

(1) $\operatorname{degree}\left(\sigma^{*} T_{E / P}\right)=d_{E}$.

(2) $P$ is maximal among all parabolic subgroups $P^{\prime}$ satisfying the condition that there is a reduction $\left(P^{\prime}, \sigma^{\prime}\right)$ of $E$ to $P^{\prime}$ such that degree $\left(\sigma^{\prime *} T_{E / P^{\prime}}\right)$ $=d_{E}$.

Then the reduction $(P, \sigma)$ is canonical.

Proof. Let $(P, \sigma)$ be a reduction of $E$ to $P$ satisfying the properties stated in the proposition. We first show that the associated Levi bundle $E_{L}$ is semistable. Suppose $E_{L}$ is not semistable. Therefore, there is a reduction of structure group $\left(P_{1}^{\prime}, \sigma_{1}^{\prime}\right)$ of $E_{L}$ to a parabolic subgroup $P_{1}^{\prime}$ of $L$ such that

$$
\operatorname{degree}\left(\sigma_{1}^{\prime *} T_{E_{L} / P_{1}^{\prime}}\right)<0 .
$$

Since the projection $P \longrightarrow L$ is a smooth map, The inverse image of $P_{1}^{\prime}$ under this is a reduced subgroup of $G$. It is easy to see that this inverse image of $P_{1}^{\prime}$ (for the projection of $P$ to $L$ ), which we will denote by $P_{1}$, is a parabolic subgroup of $G$. Indeed, the quotient $G / P_{1}$ is a fiber bundle over $G / P$ with fiber $P / P_{1} \cong L / P_{1}^{\prime}$. Hence $G / P_{1}$ is complete.

Since $P \longrightarrow L$ is surjective, it follows by Lemma 2.1 that the reduction $\left(P_{1}^{\prime}, \sigma_{1}^{\prime}\right)$ gives a reduction of structure group of the principal $P$-bundle $\sigma^{*} E$ to $P_{1}$. We will denote this principal $P_{1}$-bundle by $\sigma_{1}^{*} E$. Note that $P_{1}$ is also a reduction of structure group of the principal $G$-bundle $E$ to $P_{1} \subset G$.

Let $\mathfrak{p}_{1}$ and $\mathfrak{p}$ denote the Lie algebras of $P_{1}$ and $P$ respectively. Also let $\mathfrak{p}_{1}^{\prime}$ and $\mathfrak{l}$ be the Lie algebras of $P_{1}^{\prime}$ and $L$ respectively. We have the following exact sequence of left $P_{1}$-modules

$$
0 \longrightarrow \mathfrak{p} / \mathfrak{p}_{1} \longrightarrow \mathfrak{g} / \mathfrak{p}_{1} \longrightarrow \mathfrak{g} / \mathfrak{p} \longrightarrow 0
$$

Since $P / P_{1} \cong L / P_{1}^{\prime}$, we conclude that $\mathfrak{p} / \mathfrak{p}_{1} \cong \mathfrak{l} / \mathfrak{p}_{1}^{\prime}$ as $P_{1}$ modules.

Recall that $\sigma_{1}^{*} E$ is a reduction of structure group of $E$ to $P_{1}$. Consider the vector bundles associated to $\sigma_{1}^{*} E$ by the left $P_{1}$ modules in (3). Since $\mathfrak{p} / \mathfrak{p}_{1} \cong \mathfrak{l} / \mathfrak{p}_{1}^{\prime},(3)$ gives the exact sequence

$$
0 \longrightarrow \sigma_{1}^{\prime *} T_{E_{L} / P_{1}^{\prime}} \longrightarrow \sigma_{1}^{*} T_{E / P_{1}} \longrightarrow \sigma^{*} T_{E / P} \longrightarrow 0
$$

of vector bundles on $X$. Now using (2) we conclude that $\operatorname{degree}\left(\sigma_{1}^{*} T_{E / P_{1}}\right)=\operatorname{degree}\left(\sigma^{*} T_{E / P}\right) \operatorname{degree}\left(\sigma_{1}^{\prime *} T_{E_{L} / P_{1}^{\prime}}\right)<\operatorname{degree}\left(\sigma^{*} T_{E / P}\right)$. 
This contradicts the minimality assumption on $(P, \sigma)$ in the statement of the proposition. Therefore, $E_{L}$ must be semistable.

Now we need to check the second condition in the definition of canonical reduction.

Let $B$ be a Borel subgroup of $G$ and $T \subset B$ be a maximal torus. Let $\Delta$ be the system of simple roots. Let $I$ denote the set of simple roots defining the parabolic subgroup $P$ that contains $B$. So $I$ define the roots of the Levi factor $L$ of $P$.

Take any character $\chi$ of $P$ whose restriction to $T$ is expressed as

$$
\left.\chi\right|_{T}=\sum_{\alpha \in \Delta-I} c_{\alpha} \alpha \sum_{\beta \in I} c_{\beta} \beta
$$

with $c_{\alpha}, c_{\beta} \geq 0$. Let $Z^{0}(L) \subset T$ be the connected component of the center of $L$ containing the identity element. The characters $\beta \in I$ of $T$ have the property that $\left.\beta\right|_{Z^{0}(L)}$ is trivial. Hence we see that if $\chi$ is a nontrivial character of $P$ of the above form then $c_{\alpha}>0$ for some $\alpha \in \Delta-$ $I$ (as each $\beta$ in $I$ defines a trivial character on $P$ ). Also, some positive multiple of a character of $Z^{0}(L)$ extends to a character of $L$. Hence there are positive integers $m_{\alpha}$, for each $\alpha \in \Delta-I$, such that $\left.\left(m_{\alpha} \alpha\right)\right|_{Z^{0}(L)}$ extends to a character $\chi_{\alpha}^{\prime}$ on $L$. Note that $m_{\alpha} \alpha$ and $\chi_{\alpha}^{\prime}$ need not agree on $T$. However, since they coincide over $Z^{0}(L)$, there are rational numbers $m_{\beta, \alpha}$ such that $\chi_{\alpha}^{\prime}=m_{\alpha} \alpha \sum_{\beta \in I} m_{\beta, \alpha} \beta$ over $T$. Therefore, for each $\alpha \in \Delta-I$, there is a positive integers $n_{\alpha}$ such that $\left.\left(n_{\alpha} \alpha\right)\right|_{Z^{0}(L)}$ extends to a character $\chi_{\alpha}$ on $L$, and furthermore,

$$
\chi_{\alpha}=n_{\alpha} \alpha \sum_{\beta \in I} n_{\beta, \alpha} \beta
$$

over $T$, where $n_{\beta, \alpha} \in \mathbb{Z}$.

Let $N=\Pi_{\alpha \in \Delta-I} n_{\alpha}$. Then we have the following equality

$$
\left.N \chi\right|_{T}=\sum_{\alpha \in \Delta-I} N c_{\alpha} \alpha \sum_{\beta \in I} N c_{\beta} \beta=\left.\sum_{\alpha \in \Delta-I} c_{\alpha}^{\prime} \chi_{\alpha}\right|_{T} \sum_{\beta \in I} c_{\beta}^{\prime} \beta
$$

for some integers $c_{\beta}^{\prime}, \beta \in I$ and $c_{\alpha}^{\prime}$, where $\alpha \in \Delta-I$, such that $c_{\alpha}^{\prime}$ is a positive multiple of $c_{\alpha}$. Hence $N \chi-\sum_{\alpha \in \Delta-I} c_{\alpha} \chi_{\alpha}$ is a character of $L$ whose restriction to $Z^{0}(L)$ is trivial (being a linear combination of all $\beta \in I$ ). This implies that

$$
N \chi=\sum_{\alpha \in \Delta-I} c_{\alpha}^{\prime} \chi_{\alpha}
$$

Hence it is enough to prove the second condition of canonical reduction for characters of the form $\chi_{\alpha}$, with $\alpha \in \Delta-I$. 
Fix an element $\alpha \in \Delta-I$. Let $P_{2} \supset P$ be the parabolic subgroup of $G$ defined by the subset $I_{2}=\{\alpha\} \cup I$ of $\Delta$. Let $L_{2}$ be its Levi quotient and $P^{\prime}$ be the maximal parabolic subgroup of $L_{2}$ defined by the image of $P$ in $L_{2}$. Consider the group of all characters of $P^{\prime}$ that are trivial on the center of $L_{2}$. This group is generated by a single dominant character $w$ of $P^{\prime}$ with respect to the root system of $L_{2}$ defined by its maximal torus $T$. Now $\chi_{\alpha}$ defines a character on $P^{\prime}$ which is trivial on the center of $L_{2}$. Hence the restriction of $\chi_{\alpha}$ to $T$ can be written as $\left.\chi_{\alpha}\right|_{T}=\left.(m w)\right|_{T}$ for some integer $m$. This enables us to write

$$
\left.(m w)\right|_{T}=n_{\alpha} \alpha \sum_{\beta \in I} n_{\beta, \alpha} \beta .
$$

A dominant weight is always a nonnegative rational linear combination of simple roots. The fact that $n_{\alpha}>0$ implies that $m>0$ and $n_{\beta, \alpha} \geq 0$. Hence $\chi_{\alpha}$ is a positive multiple of $w$.

To verify the second condition of a canonical reduction, we consider the parabolic subgroup $P_{2}$ and its Levi quotient $L_{2}$ defined above. Let $E_{P}$ denote the principal $P$-bundle defined by the reduction of structure group $(P, \sigma)$ of $E$. We have already noted that $P_{2} / P \cong L_{2} / P^{\prime}$. Therefore, as in the first part of the proof of the proposition, we have the following exact sequence of vector bundles

$$
0 \longrightarrow \sigma^{*} T_{E_{L_{2}} / P^{\prime}} \longrightarrow \sigma^{*} T_{E / P} \longrightarrow \sigma_{2}^{*} T_{E / P_{2}} \longrightarrow 0
$$

over $X$, where $\sigma_{2}$ is a reduction of structure group of $E$ to $P_{2}$. The principal $P_{2}$-bundle given by the reduction $\sigma_{2}$ is $E_{P}\left(P_{2}\right)$ for the inclusion of $P$ in $P_{2}$ and $E_{L_{2}}:=E_{P}\left(P_{2}\right)\left(L_{2}\right)$ is the principal $L_{2}$-bundle for the natural projection of $P_{2}$ to $L_{2}$, and $\sigma^{\prime}$ is the reduction of structure group of $E_{L_{2}}$ to $P^{\prime}$ which is $E_{P}\left(P^{\prime}\right)$ for the obvious projection of $P$ to $P^{\prime}$.

From the above exact sequence we have

$$
\operatorname{degree}\left(\sigma^{\prime *} T_{E_{L_{2}} / P^{\prime}}\right)=\operatorname{degree}\left(\sigma^{*} T_{E / P}\right)-\operatorname{degree}\left(\sigma_{2}^{*} T_{E / P_{2}}\right) .
$$

Now the assumption that $\operatorname{degree}\left(\sigma^{*} T_{E / P}\right)=d_{E}$ gives the inequality degree $\left(\sigma^{\prime *} T_{E_{L_{2}} / P^{\prime}}\right)<0$. Now, from this it follows that degree $\left(\chi_{\alpha *}\left(\sigma^{*} E\right)\right)>$ 0 . Indeed, the top exterior product of $\sigma^{\prime *} T_{E_{L_{2}} / P^{\prime}}$, namely $\operatorname{det}\left(\sigma^{\prime *} T_{E_{L_{2}} / P^{\prime}}\right)$, is the line bundle associated to the $P^{\prime}$-bundle $E_{P}\left(P^{\prime}\right)$ for the character of $P^{\prime}$ which is a negative multiple of $w$. Hence it follows from the above that some positive powers of $\chi_{\alpha *}\left(\sigma^{*} E\right)^{*}$ and $\operatorname{det}\left(\sigma^{\prime *} T_{E_{L_{2}} / P^{\prime}}\right)$ coincide, proving that degree $\left(\chi_{\alpha_{*}}\left(\sigma^{*} E\right)\right)>0$. Therefore, the second condition in the definition of canonical reduction holds. This completes the proof of the proposition. 
The above proposition establishes the existence of a canonical reduction.

Remark 3.2. Let $E_{P}$ be a principal $P$-bundle which is a reduction of structure group of $E$ satisfying the two conditions in Proposition 3.1. The proof of Proposition 3.1 shows that for any parabolic subgroup $P^{\prime}$ of $G$ containing $P$, the principal $P^{\prime}$-bundle $E_{P}\left(P^{\prime}\right)$ satisfies the second condition of a canonical reduction. Let $E_{Q}$ be any reduction of structure group of $E$ to a parabolic subgroup $Q \subset P$ of $G$ satisfying the condition $E_{Q}(P)=E_{P}$. The proof of Proposition 3.1 also shows that if the reduction $E_{Q}$ satisfies the first condition in Proposition 3.1, then it must satisfy the first condition for canonical reduction.

\section{$\S 4$. The uniqueness of canonical reduction in characteristic zero}

In this section we assume that the base field is algebraically closed of characteristic 0 . Under this assumption we will show that there is a unique canonical reduction.

The following is the main result of this section.

Theorem 4.1. Any principal G-bundle admits a unique, up to equivalence, canonical reduction. In other words, if we fix a Borel subgroup B of $G$, then there is exactly one canonical reduction to a unique parabolic subgroup containing $B$.

Proof. The existence of a canonical reduction is established in Proposition 3.1. So we only need to verify the uniqueness.

Let $(P, \sigma)$ be a canonical reduction of structure group of $E$. We will denote this principal $P$-bundle $\sigma^{*} E$ also by $E_{P}$.

Let $U$ denote the unipotent radical of $P$ and $L:=P / U$ be the Levi factor. Let $\mathfrak{p}$ and $\mathfrak{u}$ denote the Lie algebras of $P$ and $U$ respectively. The adjoint action of $P$ on $\mathfrak{p}$ preserves the sub-algebra $\mathfrak{u}$.

The principal $L$-bundle $E_{P}(L)$, which is the extension of structure group of $E_{P}$, will be denoted by $E_{L}$. Since the reduction $E_{P}$ is canonical, $E_{L}$ is semistable by definition.

The vector bundle associated to $E_{P}$ for the adjoint representation of $P$ on $\mathfrak{p}$ (respectively, $\mathfrak{g} / \mathfrak{p})$ is naturally identified with $\operatorname{ad}\left(\sigma^{*} E\right)$ (respectively, $\left.\sigma^{*} T_{E / P}\right)$.

Consider the filtrations of left $P$-modules

$$
0=V_{0} \subset V_{1} \subset \cdots \subset V_{k}=\mathfrak{g} / \mathfrak{p}
$$

and 


$$
0=V_{0}^{\prime} \subset V_{1}^{\prime} \subset \cdots \subset V_{m}^{\prime}=\mathfrak{u}
$$

such that the quotients $W_{i}:=V_{i} / V_{i-1}$ and $\left.W_{j}^{\prime}:=V_{j}^{\prime} / V_{j-1}^{\prime}\right)$ are all irreducible $P$-modules. From the assumptions on $W_{i}$ and $W_{j}^{\prime}$ it follows that $U$ acts trivially on them. In other words, the actions of $P$ on $W_{i}$ and $W_{j}^{\prime}$ factor through the quotient $L$.

Let $\mathcal{V}_{j}$ (respectively, $\mathcal{V}_{j}^{\prime}$ ) denote the vector bundle over $X$ associated to the $E_{P}$ for the left $P$-module $V_{j}$ (respectively, $\left.V_{j}^{\prime}\right)$. Therefore, $\operatorname{ad}\left(\sigma^{*} E\right)$ (respectively, $\sigma^{*} T_{E / P}$ ) is filtered by the subbundles $\mathcal{V}_{j}$ (respectively, $\mathcal{V}_{j}^{\prime}$ ).

Since $W_{i}$ and $W_{j}^{\prime}$ are all irreducible left $L$-modules and $E_{L}$ is semistable, we conclude that the vector bundles $\mathcal{W}_{j}$ and $\mathcal{W}_{j}^{\prime}$ are semistable $[8$, Theorem $3.18]$.

Let $B$ be a Borel subgroup contained in the parabolic subgroup $P$ and $T$ be a maximal torus in $B$. Let $\Delta$ denote the set of simple roots. Let $I \subset \Delta$ denote the set of simple roots defining the parabolic subgroup $P$. The weights of $T$ on $\mathfrak{g} / \mathfrak{p}$ are of the form

$$
\gamma=\sum_{\alpha \in \Delta} c_{\alpha} \alpha
$$

with $c_{\alpha} \leq 0$ and $c_{\alpha}<0$ for at least one $\alpha \in \Delta-I$. The weights of $T$ on $\mathfrak{u}$ are of the form $-\gamma$, where $\gamma$ is a weight on $\mathfrak{g} / \mathfrak{p}$.

From this it follows that the character of $P$ defined by the determinant of the representation of $P$ on $W_{j}$ (respectively, $W_{j}^{\prime}$ ) is non-trivial and is a nonpositive (respectively, nonnegative) linear combination of roots in $\Delta$. Now by the second condition in the definition of a canonical reduction we see that

$$
\operatorname{degree}\left(\mathcal{W}_{j}\right)<0
$$

(respectively, degree $\left.\left(\mathcal{W}_{j}^{\prime}\right)>0\right)$.

The adjoint action of $P$ on $\mathfrak{p} / \mathfrak{u}$ factors through its Levi factor $L$ and this is the adjoint representation of $L$. In other words, the vector bundle associated to $E_{P}$ for the left $P$-module $\mathfrak{p} / \mathfrak{u}$ is identified with the adjoint bundle $\operatorname{ad}\left(E_{L}\right)$. Since $E_{L}$ is semistable, we have $\operatorname{ad}\left(E_{L}\right)$ to be semistable. Note that the degree of $\operatorname{ad}\left(E_{L}\right)$ is zero.

Consider the exact sequence

$$
0 \longrightarrow \operatorname{ad}\left(E_{P}\right) \longrightarrow \operatorname{ad}(E) \longrightarrow \sigma^{*} T_{E / P} \longrightarrow 0 .
$$

From the above observations it follows immediately that the vector bundles $\sigma^{*} T_{E / P}$ (respectively, ad $\left.\left(E_{P}\right)\right)$ has the filtration

$$
0=\mathcal{V}_{0} \subset \mathcal{V}_{1} \subset \mathcal{V}_{2} \subset \cdots \subset \mathcal{V}_{k}=\sigma^{*} T_{E / P}
$$


such that each quotient $\mathcal{W}_{j}=\mathcal{V}_{j} / \mathcal{V}_{j-1}$ is semistable of negative degree. Similarly, we have

$$
0=\mathcal{V}_{0}^{\prime} \subset \mathcal{V}_{1}^{\prime} \subset \cdots \subset \mathcal{V}_{m}^{\prime} \subset \operatorname{ad}\left(E_{P}\right)
$$

with each quotient $\mathcal{W}_{j}^{\prime}=\mathcal{V}_{j}^{\prime} / \mathcal{V}_{j-1}^{\prime}$ semistable of positive degree and $\operatorname{ad}\left(E_{P}\right) / \mathcal{V}_{k}^{\prime}$, which is identified with ad $\left(E_{L}\right)$, is semistable of degree zero.

Let $\left(P_{1}, \sigma_{1}\right)$ and $\left(P_{2}, \sigma_{2}\right)$ be two canonical reductions of the $G$-bundle $E$. In view of the Lemma 2.2 it suffices to show that the two sub-bundles $\operatorname{ad}\left(\sigma_{1}^{*} E\right)$ and $\operatorname{ad}\left(\sigma_{2}^{*} E\right)$ of the adjoint vector bundle ad $(E)$ are the same.

Using (4) together with the above descriptions of $\operatorname{ad}\left(E_{P}\right)$, and $\sigma^{*} T_{E / P}$, it follows that the composition homomorphism

$$
\operatorname{ad}\left(\sigma_{1}^{*} E\right) \longrightarrow \operatorname{ad}(E) \longrightarrow \sigma_{2}^{*} T_{E / P}
$$

is the zero homomorphism. Indeed, this follows immediately by setting $F^{1}=\operatorname{ad}\left(\sigma_{1}^{*} E\right)$ and $F^{2}=\sigma_{2}^{*} T_{E / P}$ in the following well-known lemma.

LEMMA 4.2. Let $F^{1}$ and $F^{2}$ be two vector bundles over $X$ with filtrations

$$
0=F_{0}^{i} \subset F_{1}^{i} \subset F_{2}^{i} \subset \cdots \subset F_{m_{i}}^{i}=F^{i},
$$

where $i \in[1,2]$, such that each $F_{j}^{i} / F_{j-1}^{i}$ is semistable with $\operatorname{degree}\left(F_{j}^{1} / F_{j-1}^{1}\right)$ $\geq 0$ and $\operatorname{degree}\left(F_{j}^{2} / F_{j-1}^{2}\right)<0$ for $j \in\left[1, m_{i}\right]$. Then $H^{0}\left(X, \operatorname{Hom}\left(F^{1}, F^{2}\right)\right)$ $=0$.

Proof. If $m_{1}=1=m_{2}$ and $\psi: F^{1} \longrightarrow F^{2}$ is a nonzero homomorphism, then we have

$$
\begin{aligned}
\operatorname{degree}\left(F^{1}\right) / \operatorname{rank}\left(F^{1}\right) & \leq \operatorname{degree}\left(\phi\left(F^{1}\right)\right) / \operatorname{rank}\left(\phi\left(F^{1}\right)\right) \\
& \leq \operatorname{degree}\left(F^{2}\right) / \operatorname{rank}\left(F^{2}\right) .
\end{aligned}
$$

as $F_{1}$ and $F_{2}$ are semistable. This contradicts the condition degree $\left(F^{1}\right) /$ $\operatorname{rank}\left(F^{1}\right) \geq 0>\operatorname{degree}\left(F^{2}\right) / \operatorname{rank}\left(F^{2}\right)$ in the statement of the lemma. Now the proof of the lemma is completed by using induction on $m_{1}$ and $m_{2}$ separately.

Continuing with the proof of Theorem 4.1, since the composition homomorphism in (7) is zero, the two sub-bundles $\operatorname{ad}\left(\sigma_{1}^{*} E\right)$ and $\operatorname{ad}\left(\sigma_{1}^{*} E\right)$ of $\operatorname{ad}(E)$ coincide. This completes the proof of the theorem.

Let $(P, \sigma)$ be the canonical reduction of $E$ and 3.1. Let $P_{2}$ be a parabolic subgroup of $G$ containing $P$. The Levi factor of $P_{2}$ will be denoted by $L_{2}$ and the image of $P$ in $L_{2}$ will be denoted by $P^{\prime}$. As before, $E_{L_{2}}$ denotes the principal $L_{2}$-bundle $\sigma^{*} E\left(L_{2}\right)$ and $\left(P^{\prime}, \sigma^{\prime}\right)$ denotes the reduction $\sigma^{*} E\left(P^{\prime}\right)$ of $E_{L_{2}}$ to $P^{\prime}$. 
Proposition 4.3. The reduction $\left(P^{\prime}, \sigma^{\prime}\right)$ of $E_{L_{2}}$ is its canonical reduction.

Proof. The Levi factor of the parabolic subgroup $P^{\prime}$ of $L_{2}$, which we will denote by $L_{3}$, is the Levi factor $L$ of $P$ itself. This identifies the principal $L$-bundle $E_{L}:=\sigma^{*} E(L)$ with the principal $L_{3}$-bundle $\sigma^{\prime *} E_{L_{2}}\left(L_{3}\right)$. Therefore, the semistability condition of $E_{L}$ ensures that $\sigma^{\prime *} E_{L_{2}}\left(L_{3}\right)$ is semistable.

The second condition for canonical reduction follows from the fact that the characters of $P^{\prime}$ which are nonnegative linear combinations of simple roots of $L_{2}$ also define characters of $P$ which are non-negative linear combinations of simple roots of $G$ as the roots of $L_{2}$ with respect to $T$ are also roots of $G$ with respect to $T$. This completes the proof of the proposition.

In the next section we will see how the canonical reduction constructed in Theorem 4.1 behaves with respect to extension of structure group.

\section{$\S 5$. Extension of structure group}

Let

$$
\rho: G \longrightarrow G_{1}
$$

be a faithful homomorphism of connected reductive algebraic groups. Let $E$ be a principal $G$-bundle. We will denote the associated principal $G_{1}-$ bundle $E\left(G_{1}\right)$ by $E_{1}$. We will compare the canonical reductions of $E$ and $E_{1}$.

By $E(G)$ (respectively, $E_{1}\left(G_{1}\right)$ ) we denote the corresponding group scheme for the conjugation action of $G$ (respectively, $G_{1}$ ) on itself. Since $\rho$ in (8) is faithful, $E(G)$ is naturally identified with a subgroup scheme of $E_{1}\left(G_{1}\right)$.

Let $(P, \sigma)$ be the canonical reduction of the $G$-bundle $E$ and $\left(P_{1}, \sigma_{1}\right)$ the canonical reduction of the $G_{1}$-bundle $E_{1}$. We have the subgroup scheme $\sigma^{*} E(P)$ (respectively, $\sigma_{1}^{*} E_{1}\left(P_{1}\right)$ ) of the adjoint bundle $E(G)$ (respectively, $E_{1}\left(G_{1}\right)$ ) associated to the canonical reduction of $E$ (respectively, $E_{1}$ ). Since $E(G)$ is a subgroup scheme of $E_{1}\left(G_{1}\right)$, we have $\sigma^{*} E(P)$ as a subgroup scheme of $E_{1}\left(G_{1}\right)$.

THEOREM 5.1. With the above notation, we have $\sigma^{*} E(P)=\sigma_{1}^{*} E_{1}\left(P_{1}\right)$ $\cap E(G)$, where the intersection takes place inside $E_{1}\left(G_{1}\right)$. 
Proof. Consider the exact sequence (4) and the filtrations (5) and (6) for the canonical reductions of $E$ and $E_{1}$. Setting $F^{1}=\operatorname{ad}\left(\sigma^{*} E\right)$ and $F^{2}=\sigma_{1}^{*} T_{E_{1} / P_{1}}$ in Lemma 4.2 we conclude that the composition

$$
\operatorname{ad}\left(\sigma^{*} E\right) \longrightarrow \operatorname{ad}\left(E_{1}\right) \longrightarrow T_{E_{1} / P_{1}}
$$

vanishes. Consequently, we have $\operatorname{ad}\left(\sigma^{*} E\right) \subset \operatorname{ad}\left(\sigma_{1}^{*} E_{1}\right)$. Now by the Remark 2.3 we obtain the inclusion $\sigma^{*} E(P) \subset \sigma_{1}^{*} E_{1}\left(P_{1}\right) \cap E(G)$.

To prove the opposite inclusion, we first observe that over the generic point $K$ of $X$, the inclusion $\sigma^{*} E(P)_{K} \subset \sigma_{1}^{*} E_{1}\left(P_{1}\right)_{K} \cap E(G)_{K}$ implies that $\sigma_{1}^{*} E_{1}\left(P_{1}\right)_{K} \cap E(G)_{K}$ defines a parabolic subgroup of $E(G)_{K}$ over the generic point. But such a parabolic subgroup extends to a parabolic subgroup scheme of $E(G)$ and hence gives rise to a reduction of structure group $\sigma^{\prime}$ of $E$ to a parabolic $P^{\prime}$ of $G$ (see Section 3.5 of [8] for more details). We will show that the reduction $\left(P^{\prime}, \sigma^{\prime}\right)$ of $E$ is canonical. This would complete the proof by uniqueness in Theorem 4.1.

To prove that the reduction $\left(P^{\prime}, \sigma^{\prime}\right)$ is canonical, first observe that the inclusion

$$
\sigma^{*} E(P) \subset \sigma^{\prime *} E\left(P^{\prime}\right)=\sigma_{1}^{*} E_{1}\left(P_{1}\right) \cap E(G),
$$

the fact that the normalizer of a parabolic is itself, and Lemma 2.4 together imply that

(1) the above parabolic subgroup $P^{\prime}$ can be so chosen that it contains $P$;

(2) the principal $P^{\prime}$-bundle $\sigma^{\prime *} E$ is identified with the extension $\sigma^{*} E(P)$ of $(P, \sigma)$ by the inclusion of $P$ in $P^{\prime}$.

Since $P \subset P^{\prime}$, from Remark 3.2 it follows that the reduction $\left(P^{\prime}, \sigma^{\prime}\right)$ satisfies the second condition for canonical reduction.

Let $U^{\prime}$ be the unipotent radical of $P^{\prime}$ and $L^{\prime}:=P^{\prime} / U^{\prime}$ the Levi factor. From (9) we have $P^{\prime}=P_{1} \cap G$. Hence it follows that $U^{\prime}=U_{1} \cap G$, where $U_{1}$ is the unipotent radical of $P_{1}$. So the inclusion of $P^{\prime}$ in $P_{1}$ induces an injective homomorphism $\rho$ of $L^{\prime}$ to $L_{1}$.

Therefore, the principal $L_{1}$-bundle $\sigma_{1}^{*} E\left(L_{1}\right)$ coincides with the extension of the principal $L^{\prime}$-bundle $\sigma^{\prime *} E\left(L^{\prime}\right)$ by the homomorphism $\rho$. Consequently, $\operatorname{ad}\left(\sigma^{* *} E\left(L^{\prime}\right)\right)$ is a sub-bundle of $\operatorname{ad}\left(\sigma_{1}^{*} E\left(L_{1}\right)\right)$. Since $\operatorname{ad}\left(\sigma_{1}^{*} E\left(L_{1}\right)\right)$ is a semistable vector bundle of degree zero and $\operatorname{ad}\left(E_{L^{\prime}}\right)$ is its sub-bundle of degree zero, it follows immediately that $\operatorname{ad}\left(\sigma^{\prime *} E\left(L^{\prime}\right)\right)$ must be semistable. Hence $E_{L^{\prime}}$ is semistable by Lemma 2.5. Therefore, the reduction $\left(P^{\prime}, \sigma^{\prime}\right)$ is canonical. We already noted that this completes the proof of the theorem. 
Remark 5.2. Theorem 5.1 implies that the canonical reduction of $E$ is determined by the canonical reduction of $E_{1}$. This is because the group scheme $\sigma^{*} E(P)$ determines the reduction $\sigma$ (Lemma 2.2).

The following proposition relates semistability of $E$ and $E_{1}$.

Proposition 5.3. If $E_{1}$ is semistable, then $E$ is semistable. In the converse direction, if the image of $G$ by the homomorphism $\rho$ in (8) is not contained in any proper parabolic subgroup of $G_{1}$, then semistability of $E$ implies that $E_{1}$ is semistable.

Proof. If $E_{1}$ is semistable, then $\sigma_{1}^{*} E_{1}\left(P_{1}\right)=E_{1}\left(G_{1}\right)$. Hence

$$
\sigma^{*} E(P)=\sigma_{1}^{*} E_{1}\left(P_{1}\right) \cap E(G)=E(G) .
$$

In other words, $E$ is semistable.

Now assume that $E$ is semistable. So, $P=G$ and $\sigma^{*} E(P)=E(G)$. If the image of $G$ is not contained in any proper parabolic subgroup of $G_{1}$, then the equality $E(G)=\sigma_{1}^{*} E_{1}\left(P_{1}\right) \cap E(G)$ implies that $P_{1}=G_{1}$. In other words, $E_{1}$ is semistable. This completes the proof of the proposition.

Remark 5.4. The above proposition is not valid without the assumption that no proper parabolic subgroup of $G_{1}$ contains the image of $G$. For example, take $G=k^{*}, G_{1}=\operatorname{SL}(2, k)$ and $\rho$ to be homomorphism that sends any $c \in k^{*}$ to the diagonal matrix with entries $c$ and $c^{-1}$. Take the $G$-bundle $E$ to be a line bundle $\xi$ of positive degree. Then $E_{1}$ corresponds to the vector bundle $\xi \oplus \xi^{*}$, which is not semistable.

\section{$\S 6$. The case of positive characteristic}

We will now indicate how the results in the previous two sections can be extended to positive characteristics. In this section $k$ will be an algebraically closed field of characteristic $p>0$.

We first recall the definition of height of a representation. Let $G$ be a connected reductive algebraic group over $k$. Let $T$ be a maximal torus of $G$ and $B$ be a Borel subgroup containing $T$. Let $R$ be the root system obtained from these choices of datum. Let $\chi^{*}(T)$ denote the character group of $T$ and $\chi_{*}(T)$ be the cocharacter group of $T$. The natural pairing $(.,):. \chi^{*}(T) \times \chi_{*}(T) \longrightarrow \mathbb{Z}$ gives us coroots $\alpha^{\vee}$ associated to roots $\alpha$. Following [11] and [5], the height of a weight $\lambda \in \chi^{*}(T)$ is defined as

$$
\operatorname{ht}_{G}(\lambda):=\sum_{\alpha>0}\left(\lambda, \alpha^{\vee}\right) .
$$


For a finite dimensional rational representation $V$ of $G$, recall the definition of height

$$
\operatorname{ht}_{G}(V):=\sup \operatorname{ht}_{G}(\lambda),
$$

where the supremum is taken over all weights $\lambda$ of $T$ in $V$. A representation $V$ of $G$ is said to be of low height if the inequality $h_{G}(V)<p$ is valid.

One observes that the above described notion of height of a representation depends only on the group $G$ and the representation $V$. In particular, it is independent of the choices of both $B$ and $T$.

We put down some properties of the height of a representation which will be useful for our purpose.

LEMMA 6.1. If $W$ is a sub-quotient of $V$ as a representation of $G$, then $\operatorname{ht}_{G}(W) \leq \operatorname{ht}_{G}(V)$.

Proof. This is obvious from the definition.

Let $G^{u}$ denote the closed subvariety of $G$ consisting of all unipotent elements and let $\mathfrak{g}_{\text {nilp }}$ be the closed subvariety of $\mathfrak{g}$ defined by its nilpotent elements.

Let $\beta^{\vee}$ denote the highest coroot of $G$ and $h=\left(\rho_{0}, \beta^{\vee}\right)$ denote the Coxeter number of $G$, where $\rho_{0}$ is half the sum of positive roots.

We now recall a theorem of Serre [11, p. 21].

TheOREM 6.2. Assume that $p>h$, where $h$ is the Coxeter number defined above. There exists a unique isomorphism of varieties $\log : G^{u} \longrightarrow$ $\mathfrak{g}_{\text {nilp }}$ with the following properties:

(1) the equality $\log (\sigma u)=\sigma \log (u)$ is valid for all $\sigma \in \operatorname{Aut}(G)$.

(2) the restriction of $\log$ to $U(k)$ defines an isomorphism of algebraic groups $U \longrightarrow \mathfrak{u}$, whose tangent map is identity.

(3) the equality $\log \left(x_{\alpha}(\theta)\right)=\theta X_{\alpha}$ is valid for every root $\alpha$ and $\theta \in k$.

Using the above theorem the inverse of the isomorphism log will be denoted by exp. For $x \in G^{u}(k)$ and $t \in k$, we define the element $x^{t}$ as $\exp (t \log (x))$.

A closed subgroup $\Gamma$ of $G$ is called saturated if for all $x \in \Gamma \cap G^{u}$, we have $x^{t} \in \Gamma$ for all $t \in k$.

Let $P$ be a parabolic subgroup of $G$. Let $U$ be the unipotent radical of $P$ and $L=P / U$ the Levi factor. We will fix a splitting $i$ of the projection 
homomorphism $P \longrightarrow L$, that is, the composition of $i$ with the projection is the identity map of $L$. So $i(L)$ is a subgroup of $P$ and hence also a subgroup of $G$.

The height of a representation does not behave well under the restriction of the representation. The following lemma due to Serre [11] (Corollary 1 of Theorem 5 therein) addresses the case when we are restricting ourselves to a saturated reductive subgroup.

Lemma 6.3. Let $p>h$. Suppose $V$ is a rational representation of $G$. Let $H \subset G$ be a saturated reductive subgroup of $G$. Then we have $\operatorname{ht}_{H}(V) \leq \operatorname{ht}_{G}(V)$.

This lemma of [11] has the following corollary:

Corollary 6.4. Let $p>h$. Suppose $V$ is a rational representation of $G$. Let $i(L) \subset G$ be a Levi subgroup of a parabolic $P$. Then we have $\operatorname{ht}_{i(L)}(V) \leq \operatorname{ht}_{G}(V)$.

Proof. In view of the above lemma we only need to check that any Levi subgroup is saturated.

Since Levi subgroups are the centralizers of a tori in $G$, it suffices to check that the centralizer of any subgroup is saturated. The saturatedness of the centralizer of a subgroup evidently follows from the fact that if $x$ is an unipotent element of $G$ and $y x y^{-1}=x$, then $y x^{t} y^{-1}=x^{t}$ (see (i) of Theorem 6.2). This completes the proof of the corollary.

Remark 6.5. One observes that the condition $p>h$ is automatically satisfied when the adjoint representation of $G$ is of low height.

The following lemma due to Ilangovan, Mehta and Parameswaran (see Lemma 3.2 of [5]) relates the notion of the height of a representation with the geometry of $G$.

Lemma 6.6. Let $V$ be a linear representation of $G$ of low height. Let $X \in \mathfrak{g}$ be a nilpotent element. Let $W \subset V$ be a vector subspace of $V$ such that $X$ belongs to the Lie algebra of the stabilizer of $W$. Then $X$ belongs to the tangent space of the reduced stabilizer.

Let $X$ be a smooth connected projective curve over an algebraically closed field $k$ of characteristic $p$. Let $E$ be a principal $G$-bundle on $X$, where $G$ is a connected reductive linear algebraic group over $k$. We will prove a proposition on the semistability of the associated vector bundles. For that purpose we will need the following lemma, which is well-known. 
Lemma 6.7. Let $H$ is a subgroup scheme of $G$ which is contained in the center of $G$. A principal $G$-bundle $E$ over $X$ is semistable if an only if the principal $G / H$-bundle $E(G / H)$ over $X$ is semistable.

We will also need the following lemma.

Lemma 6.8. Assume that the adjoint representation of $G$ is of low height. Let $p: X^{\prime} \longrightarrow X$ be a finite separable morphism. A principal $G$-bundle $E$ over $X$ is semistable if and only if the pulled back $G$-bundle $p^{*} E$ over $X^{\prime}$ is semistable.

Proof. If $p^{*} E$ is semistable, then $E$ is evidently semistable. Indeed, otherwise the pullback of any destabilizing reduction of $E$ destabilizes $p^{*} E$.

For the converse, [5, Theorem 3.1] implies that the adjoint bundle ad $(E)$ is semistable. (The Lemma 3.1 of [5] is recalled below in the proof of Proposition 6.9.) Hence the vector bundle $p^{*} \operatorname{ad}(E)=\operatorname{ad}\left(p^{*} E\right)$ is semistable. But the semistability of $\operatorname{ad}\left(p^{*} E\right)$ implies that the $G$-bundle $p^{*} E$ is semistable. This completes the proof of the lemma.

Proposition 6.9. Let $G$ be a connected reductive linear algebraic group over $k$ such that the adjoint representation of $G$ is of low height. Let $\rho: G \longrightarrow \mathrm{GL}(V)$ be a linear representation of low height such that the connected component of the center of $G$ maps to the connected component of the center of $\mathrm{GL}(V)$. If $E$ is a semistable principal $G$-bundle over $X$ then the vector bundle $\rho_{*} E$ is semistable.

Proof. The above proposition is proved in [5] under the assumption that $G$ is semisimple (see [5, Theorem 3.1] which is the above proposition with the extra assumption that $G$ is semisimple). We will show how to extend this to the more general case of reductive groups.

Let $G_{0}$ denote the semisimple group $G / Z^{0}(G)$, where $Z^{0}(G)$ is the connected component of the center of $G$ containing the identity element. Then the condition in the statement of proposition implies that the representation $\rho$ factors through a homomorphism $\rho_{0}: G_{0} \longrightarrow \operatorname{PGL}(V)$.

By setting $G_{1}=[G, G]$, and restriction of $\rho$ to $G_{1}$, we obtain a homomorphism $\rho_{1}: G_{1} \longrightarrow \mathrm{SL}(V)$. Let

$$
j: G_{1} \longrightarrow G_{0}
$$

be the surjective homomorphism (in the flat topology) obtained by restricting the quotient map of $G$ to $G_{0}$. 
We have the $G_{0}$-bundle $E_{0}:=E\left(G_{0}\right)$, which is semistable by Lemma 6.7 and Lemma 6.8. To complete the proof of the proposition it suffices to show that the PGL $(V)$-bundle $\rho_{0 *} E_{0}$ is semistable. To prove that $\rho_{0 *} E_{0}$ is semistable, we consider a finite separable morphism

$$
p: X^{\prime} \longrightarrow X
$$

such that $p^{*} E_{0}=j_{*} E_{1}^{\prime}$ for some principal $G_{1}$-bundle $E_{1}^{\prime}$ over $X^{\prime}$. Therefore, using Lemma 6.7 and Lemma 6.8 it follows that $E_{1}^{\prime}$ is semistable.

Since the maximal torus $T$ of $G$ can be written as $T_{1} \cdot Z^{0}(G)$, we see that the weights of the representation $\rho_{1}$ are restrictions of the weights of the representation $\rho$, and furthermore, the coroots of $G_{1}$ coincide with those of $G$. Consequently, the representation $\rho_{1}$ is of low height. Now it follows from [5, Theorem 3.1] that the $\mathrm{SL}(V)$ bundle $\rho_{1 *} E_{1}^{\prime}$ over $X^{\prime}$ is semistable. So Lemma 6.7 implies that $p^{*} \rho_{0 *} E_{0}$ is semistable. Finally, applying Lemma 6.8 to $\rho_{0 *} E_{0}$ the proof of the proposition is completed.

We now return to the question on canonical reduction of $E$. In Proposition 3.1 we proved that a canonical reduction exists. Now, given a reduction $(P, \sigma)$ of $E$, note that there is an exact sequence

$$
0 \longrightarrow \operatorname{ad}\left(\sigma^{*} E\right) \longrightarrow \operatorname{ad}(E) \longrightarrow \sigma^{*} T_{E / P} \longrightarrow 0
$$

of vector bundles. The following proposition describes the canonical reduction of $E$.

Proposition 6.10. Let $(P, \sigma)$ be a canonical reduction. Suppose that the adjoint representation of $G$ is of low height. Then we have filtrations

$$
0=\mathcal{V}_{0} \subset \mathcal{V}_{1} \subset \cdots \subset \mathcal{V}_{k}=\sigma^{*} T_{E / P}
$$

(respectively, $0=\mathcal{V}_{0}^{\prime} \subset \mathcal{V}_{1}^{\prime} \subset \cdots \subset \mathcal{V}_{k}^{\prime} \subset \operatorname{ad}\left(\sigma^{*} E\right)$ ) such that each quotient $\mathcal{W}_{j}:=\mathcal{V}_{j} / \mathcal{V}_{j-1}$ (respectively, $\left.\mathcal{W}_{j}^{\prime}:=\mathcal{V}_{j}^{\prime} / \mathcal{V}_{j-1}^{\prime}\right), j \in[1, k]$, is semistable of negative (respectively, positive) degree and $\operatorname{ad}\left(E_{L}\right)=\operatorname{ad}\left(\sigma^{*} E\right) / \mathcal{V}_{k}^{\prime}$ is semistable of degree zero. Here $E_{L}$ is the extension of structure group of the $P$-bundle $E_{P}$, defined by $\sigma$, to the Levi factor $L$ of $P$.

Proof. The main idea of the proof is already there in Theorem 4.1. We need to check that under the present hypothesis the proof goes through in characteristic $p$.

The assumption in the statement of the theorem ensures that for the adjoint representation of $G$ on $\mathfrak{g}$ we have $\operatorname{ht}_{G}(\mathfrak{g})<p$. Let $(P, \sigma)$ be a 
canonical reduction. Let $L$ be the Levi quotient. Let $i$ be a splitting of $P \longrightarrow L$. So, we will denote by $i(L)$ the Levi subgroup of $P$.

Now by Lemma 6.4, Lemma 6.1 and Remark 6.5 we have $\mathrm{ht}_{i(L)}(W)<p$ for every representation $W$ of $i(L)$ which is a sub-quotient of $\mathfrak{g}$.

The vector bundles $\mathcal{W}_{j}, \mathcal{W}_{j}^{\prime}$, and adjoint vector bundle $\operatorname{ad}\left(E_{L}\right)$ are associated to $E_{L}$ by the representations of $L$ on the sub-quotients $W_{j}, W_{j}^{\prime}$ and the Lie algebra $\mathfrak{l}$ ( of $L$ ) respectively. The proof that these vector bundles are semistable is now a consequence of Proposition 6.9 once we check that these representations of $L$ are of low height. But these representations coincide with the representations of $i(L)$ on these sub-quotients of $\mathfrak{g}$. Hence the proof of the proposition is complete.

Now we state some corollaries.

COROLLARY 6.11. If the adjoint representation of $G$ is of low height, then the canonical reduction exists and it is unique.

Proof. We just imitate the proof of Theorem 4.1. It is straight-forward to check that the proof goes through.

The following statement without the assumption on the low height was conjectured by Behrend [3].

COROLlary 6.12. If the adjoint representation of $G$ is of low height then if $(P, \sigma)$ is a canonical reduction, then we have $H^{0}\left(X, \sigma^{*} T_{E / P}\right)=0$.

Proof. It follows immediately from the fact that if $V$ is a vector bundle on $X$ which admits a filtration by sub bundles, whose successive quotients are semistable of negative degree then $H^{0}(X, V)=0$.

We now return to the characteristic $p$ version of Theorem 5.1.

Let $\rho: G \longrightarrow \mathrm{GL}(V)$ be a faithful homomorphism of connected reductive algebraic groups. Let $E$ be a principal $G$-bundle over $X$. We will denote the associated principal $\mathrm{GL}(V)$-bundle by $E_{1}$. Let $(P, \sigma)$ be the canonical reduction of $E$. Let $\left(P_{1}, \sigma_{1}\right)$ be the canonical reduction of $E_{1}$. By $E(G)$ and $E_{1}(\mathrm{GL}(V))$ we denote the group schemes associated to the actions of $G$ and $\mathrm{GL}(V)$ on themselves by inner conjugation. Also we have the sub group schemes $\sigma^{*} E(P)$ and $\sigma_{1}^{*} E_{1}\left(P_{1}\right)$, of $E(G)$ and $E_{1}(\mathrm{GL}(V))$ respectively, associated to the canonical reductions. 
THEOREM 6.13. Suppose that the adjoint representations of both $G$ and $\mathrm{GL}(V)$ are of low height and $\rho$ is also of low height. Then we have $\sigma^{*} E(P)=\sigma_{1}^{*} E_{1}\left(P_{1}\right) \cap E(G)$.

Proof. If the adjoint representations are of low height then the first part of the proof of Theorem 5.1 goes through. Hence we get an inclusion $\sigma^{*} E(P) \subset \sigma_{1}^{*} E_{1}\left(P_{1}\right) \cap E(G)$. The proof of the opposite inclusion evidently remains valid if we make the following assumption: if $K$ is the generic point of $X$ then $\sigma_{1}^{*} E_{1}\left(P_{1}\right)_{K} \cap E(G)_{K}$ defines a parabolic subgroup of $E(G)_{K}$. We claim that under the low height assumption this indeed holds. This would prove the theorem.

To prove the claim we need to verify that

$$
\operatorname{Lie}\left(\sigma_{1}^{*} E_{1}\left(P_{1}\right)_{K}\right) \cap\left(E(G)_{K}\right)=\operatorname{Lie}\left(\left(\sigma_{1}^{*} E_{1}\left(P_{1}\right)_{K} \cap E(G)_{K}\right)_{\text {red }}\right) .
$$

It suffices to verify the last statement after the base change to the algebraic closure $\bar{K}$ of $K$. Note that the above algebraic groups over $K$ once base changed to $\bar{K}$ are of the form $G \times_{k} \bar{K}$ and $\operatorname{GL}(V) \times_{k} \bar{K}$. Therefore, the question reduces to the following proposition.

Proposition 6.14. Let $\rho: G \longrightarrow \mathrm{GL}(V)$ be a low height representation of a reductive algebraic group over an algebraically closed field $\bar{K}$. Let $P$ and $P_{1}$ be parabolic subgroups of $G$ and $\mathrm{GL}(V)$ respectively such that $P \subset P_{1} \cap G=P^{\prime}$. Then $P^{\prime}$ is a parabolic subgroup of $G$.

Proof. If $P_{1}$ is a maximal parabolic subgroup, then the proposition is just Lemma 6.6. The parabolic $P_{1}$ can in general be written as an intersection of finitely many maximal parabolic subgroups $P_{1}^{1}, \cdots, P_{1}^{k}$. Since all of these maximal parabolic subgroups contain the parabolic $P$, It follows from Lemma 6.6 that $P_{1}^{j} \cap G$ are already reduced. But

$$
P_{1} \cap G=\bigcap_{j \in[1, k]}\left(P_{1}^{j} \cap G\right) .
$$

This implies that $P_{1} \cap G$ is an intersection of a finitely many parabolic subgroups containing a parabolic $P$, and hence is a parabolic subgroup. This completes the proof of the proposition.

Using Proposition 6.14, the proof of Theorem 6.13 follows just by repeating the steps in the proof of Theorem 5.1. 


\title{
REFERENCES
}

[1] M. F. Atiyah and R. Bott, The Yang-Mills equations over Riemann surfaces, Phil. Trans. Roy. Soc. Lond. A, 308 (1982), 523-615.

[2] B. Anchouche, H. Azad and I. Biswas, Harder-Narasimhan reduction for principal bundles over a compact Kähler manifold, Math. Ann., 323 (2002), 693-712.

[3] K. A. Behrend, Semistability of reductive group schemes over curves, Math. Ann., 301 (1995), 281-305.

[4] G. Harder and M. S. Narasimhan, On the cohomology groups of moduli spaces of vector bundles on curves, Math. Ann., 212 (1975), 215-248.

[5] S. Ilangovan, V. B. Mehta and A. J. Parameswaran, Semistability and semisimplicity in representations of low height in positive characteristic, A tribute to C. S. Seshadri, a collection of articles on Geometry and Representation Theory, to appear.

[6] S. Kumar and M. S. Narasimhan, Picard group of the moduli spaces of G-bundles, Math. Ann., 308 (1997), 155-173.

[7] V. B. Mehta and S. Subramanian, On the Harder-Narasimhan filtration of principal bundles, Algebra, Arithmetic and Geometry (Ed. R. Parimala), pp. 405-415. Narosa Pub. House, 2002.

[8] S. Ramanan and A. Ramanathan, Some remarks on the instability flag, Tohoku Math. J., 36 (1984), 269-291.

[9] A. Ramanathan, Moduli of principal bundle, Algebraic geometry (Proc. Summer Meeting, Univ. Copenhagen, 1978), pp. 527-533, Lecture Notes in Math. 732, Springer, Berlin, 1979.

[10] J.-P. Serre, Sur la semisimplicité des produits tensorial de représentations, Invent. Math., 116 (1994), 513-530.

[11] J.-P. Serre, Moursund Lectures, (notes by W. E. Duckworth). University of Oregon Mathematics Department, 1998.

\author{
Indranil Biswas \\ School of Mathematics \\ Tata Institute of Fundamental Research \\ Homi Bhabha Road \\ Bombay 400005, India \\ indranil@math.tifr.res.in \\ Yogish I. Holla \\ School of Mathematics \\ Tata Institute of Fundamental Research \\ Homi Bhabha Road \\ Bombay 400005, India \\ yogi@math.tifr.res.in
}

